\title{
An unusual cause of intractable vomiting after cisplatin chemotherapy
}

A 59-year-old woman with advanced cervical carcinoma presented to us with intractable vomiting, 8 weeks after receiving cisplatin chemotherapy. Upper gastrointestinal endoscopy revealed mild duodenitis and an obstructive adhesion in the distal duodenum (Figure 1 ) The adhesion was disrupted by the passage of the endoscope and the patient's symptoms promptly resolved.

Endoscopy_UCTN_Code_CCL_1AB_2AZ_3AC
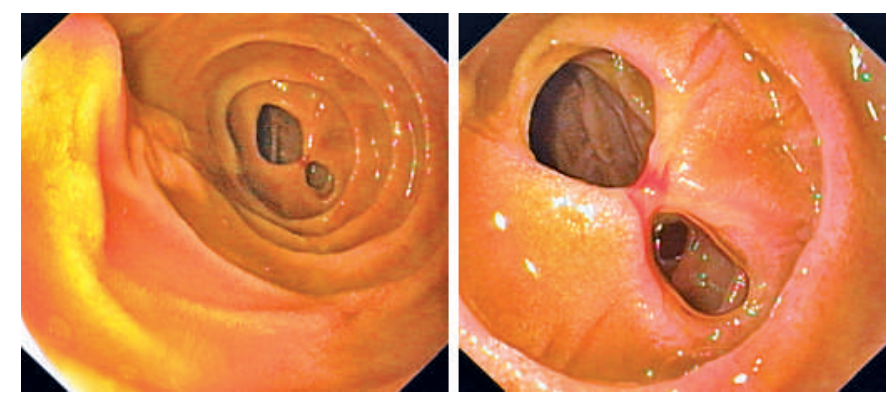

Figure 1 Endoscopic views of the obstructive duodenal adhesion at the distal end of the descending part of the duodenum that had formed secondary to duodenitis.

Corresponding Author

\section{Storr, M.D.}

Department of Medicine II

Klinikum Grosshadern

Ludwig-Maximilians-University of

Munich

Marchioninistraße 15

81377 Munich

Germany

Fax: +49-89-7095-5281

E-mail: martin.storr@med.

uni-muenchen.de 